\title{
Rhétorique de la Covid-19: analyse de trois cas de paroles « infectées »
}

\begin{abstract}
Aboubakar GOUNOUGO ${ }^{1}$
Abstract

The irruption of Covid-19 in the public space has contributed to perverting or infecting speech and its commerce between social actors. By perversion or infection of speech, we mean this polemical discourse, bordering on belligerence, which confronts men, all troubled by the emerging pandemic. Speeches made in times of Covid-19 crisis are nihilistic in that they convey contempt, hatred, mistrust, suspicion or conspiracy, fear, etc. between men and nations. Three of these discourses summarize the genre here. These are the formula "the Chinese virus" of Donald Trump, the exchange between the doctors Jean-Paul Mira and Camille Cocht and the conference of the pan-Africanist leader Kemi Seba, about the vaccination against the Covid. The objective of this contribution is to analyze these three cases of socio-political discourse to highlight the conflict of their respective dialogisms.
\end{abstract}

Keywords: Covid-19; public space; infection; speech; conflict

DOI: $10.24818 / \mathrm{DLG} / 2021 / 38 / 03$

\section{Introduction}

7 Dout énoncé, quelle que soit sa tonalité, met en relation un locuteur et un interlocuteur autour d'un objet du sens ou objet du message. En un mot, tout énoncé a une valeur pragmatique. Selon la conception de T. Todorov et O. Ducrot, la pragmatique décrit, «l'usage que peuvent faire des formules, des interlocuteurs visant à agir les uns sur les autres » (1972 : 423). Parmi ces formules qui fondent la parole partagée par les locuteurs dans des interactions dialogiques, il en existe certaines qui intéressent les analystes du discours et d'autres spécialistes des sciences humaines $\mathrm{du}$ fait de la tension, mieux de $\mathrm{l}^{\prime}$ « hypertension » qu'elles provoquent dans les échanges discursifs. Ce sont les formules à valeur polémique ou conflictuelle ou toutes ces formes d'agressions verbales susceptibles de circuler dans les interlocutions. Dans la volonté pragmatique des locuteurs d'agir les uns sur les autres, ce qui est la preuve,

1 Aboubakar Gounougo, Université Félix Houphouët-Boigny, Côte d'Ivoire, degounougo@yahoo.fr 
selon J. Moeschler et A. Reboul, que « l'usage du langage (...) n'est neutre, dans ses effets, ni sur le processus de communication, ni sur le système linguistique lui-même » (1994:17), il ressort que de nombreuses paroles à visée polémique, du point de vue de leur fonction illocutoire et perlocutoire, sont le plus souvent graves et lourdes de conséquences. Dans le contexte très dysphorique de la pandémie de la Covid-19 dont le surgissement dans le monde a provoqué une onde de choc, des paroles offensantes, voire belliqueuses sont proférées de-ci de-là, sans pour autant que les personnes qui les profèrent n'ignorent l'impact qu'elles peuvent avoir sur leurs récepteurs. C'est dire que cette crise sanitaire s'est non seulement attaquée à l'intégrité physique de l'homme mais également à tous les fondamentaux qui garantissent sa présence au monde. L'un de ces fondamentaux est la pratique de la parole dont la nécessité est rapportée dans ces propos de P. Breton : « la parole est ce qui nous lie aux autres, que nous nous adressons à eux directement ou par le biais d'outils de communication divers. Elle est aussi ce qui nous lie à nous-même : nous ne cessons en effet de nous parler » $(2007: 5)$.

Sous l'effet de la panique générale engendrée par la pandémie du coronavirus, la parole a perdu chez certains sa valeur irénique pour ne plus être utilisée qu'à des fins polémiques et conflictuelles qui, elles-mêmes, servent, entre autres des desseins politiques, racistes et idéologiques. À la recherche de boucs émissaires pour couvrir son incapacité à juguler et éradiquer la pandémie qui fait des ravages, la société se dresse contre ellemême. Et c'est la parole qui est utilisée par les uns et les autres pour régler leurs comptes. Elle devient de fait un facteur de désocialisation et de conflit. Dit autrement, la parole s'est « infectée ». En ce sens, des ethos, qui fabriquent cette parole pour leurs besoins de communication, lui inoculent des formules qui déstabilisent nécessairement la réception et la rendent dysphorique. Le logos qui fonde donc ces formules en ce temps de Covid-19 est polémique, conflictuel et c'est à juste titre qu'il implante des antagonismes quasi irréductibles entre énonciateurs et énonciataires dans les interactions dialogiques qui les lient, directement ou indirectement.

Cela dit, pour la conduite de notre réflexion, nous nous sommes interrogés sur les faits inhérents à trois cas de parole pour comprendre en quoi elles sont infectées. Ce sont : la formule « virus chinois » de Trump, de la parole partagée par deux spécialistes de médecine sur la chaîne LCI et de celle proférée par l'activiste panafricaniste Kemi Seba. Comment fonctionnent ces paroles et en quoi la conflictualité est-elle la qualité 
première de leurs dialogismes ? La réponse à cette préoccupation sera ici le résultat d'une analyse de quelques discours relevant des domaines politique, médical et social dans lesquels se recensent des exemples de cette parole infectée, c'est-à-dire offensante et polémique. Une parole qui est la preuve des rapports dialogiques tendus entre acteurs du monde, tourmentés par les vagues mortelles du coronavirus. Dans le choix de cette méthode d'analyse, nous ne passerons pas par pertes et profits les ressources des analyses rhétorique et pragma-énonciative qui donnent du coffre à toute analyse de discours. Ces deux méthodes d'analyse du discours ont en commun de fournir un outillage épistémologique permettant de décrire les interactions dialogiques pour en ressortir la nature, le fonctionnement et la portée pragmatique. De façon concrète, nous sonderons la «dimension médiologique $»^{2}$ de l'internet dans ce qu'elle offre d'articles de presse en ligne, de contributions libres sur la pandémie, de vidéos de cyber activistes professionnels ou amateurs en la matière, d'interviews, etc., qui sont autant de discours dans lesquels nous relevons des formules «polluant» la parole au point de ne plus la rendre éthique. Pour ce qui est de notre corpus, il est composé des discours de Trump, du dialogue entre les médecins Jean-Paul Mira et Camille Cocht et de la conférence du leader panafricaniste Kemi Seba, que nous avons pris sur des pages numériques et sur YouTube, le 12 septembre 2020. Nous avons transcrit les paroles des vidéos pour obtenir nos énoncés et pour chacun de ces énoncés cités, les références sont à retrouver en notes infrapaginales, dans le corps de notre analyse. Ainsi, sur la base de ces discours, nous allons articuler notre réflexion en trois grands points relatifs à l'analyse des propos de Trump sur le coronavirus qu'il nomme le «virus chinois », de la parole partagée par les deux spécialistes de médecine sur la chaîne LCI et de la parole proférée par l'activiste panafricaniste Kemi Seba.

\section{2. «Le virus chinois » : une formule polémique pour brouiller les pistes}

La Covid-19 a provoqué de grandes tensions dans certaines sphères de la vie publique, à commencer par la sphère politique, du fait des propos allusifs tenus par certains acteurs de premier plan. La panique devant la

\footnotetext{
${ }^{2}$ Ce concept employé par Maingueneau (2002: 14) pour désigner le mode d'existence matériel du texte qui ne fait «qu'un avec son mode de support /transport et de stockage, donc de mémorisation ».
} 
pandémie émergente et inexpliquée et l'incapacité des politiciens à trouver des solutions idoines pour sortir leurs peuples de la tragédie sanitaire ont poussé certains d'entre eux à opter pour des postures et stratégies antipathiques et conflictuelles. Un cas qui illustre l'énonciation politique à caractère polémique et discourtois est celui du Président américain Donald Trump qui a taxé le coronavirus de « virus chinois » :

Nous continuons nos efforts acharnés pour venir à bout du virus chinois ${ }^{3}$.

Dans cette formule de Trump, l'implicite, précisément le sousentendu est la stratégie argumentative du locuteur. Selon Ruth Amossy, le sous-entendu "dérive du fait qu'il permet au locuteur à la fois de "dire certaines choses, et de pouvoir faire comme si on ne les avait pas dites" » (2012: 191) et que de ce fait il "engage l'allocutaire à compléter les éléments manquants» (Ibid.:190). Tous ceux qui ont entendu ou lu ce propos du Président américain ont saisi ses non-dits. Ceux-ci, une fois interrogés, révèlent que la formule de Trump ne relève pas seulement de la polémique, mais aussi d'autre chose si nous entendons cette praxis sociale dans le même sens que Daciana Vlad. Cette dernière distingue la polémique de toutes celles qui partagent avec elle certains traits de ressemblance à partir d'une étude componentielle qui contient ces propos :

la polémique, le débat ou la controverse, échanges (...) se caractérisent par des traits comme [+ activité intellectuelle], [+caractère public], [+ civilité], [+ argumentativité forte], [+ durée], [et] s'opposent à la chamaillerie, au démêlé, à la prise de bec, à l'altercation, à l'engueulade, ou à l'empoignade, qui partagent des caractéristiques telles que [- activité intellectuelle], [+ caractère privé], [- civilité], [+ argumentativité faible], [- durée] (2010:1).

Sur la base de cette proposition de Vlad, il ressort que la formule «virus chinois » proférée par le Président américain à destination de ses récepteurs est tendancieuse. Elle contient les mêmes traits sémiques que toutes ces formes conflictuelles évoquées par Vlad. Ainsi, cette formule de

3 Consulter les sources: https://www.liberation.fr/direct/element/quand-trumpcorrige-son-discours-a-la-main-pour-dire-virus-chinois-plutot-quecoronavirus_110962/ et: https://www.letemps.ch/opinions/nom-virus-quil-cache https://www.youtube.com/watch?v=CRKJzGenzHY 
Trump qui est employée obsessionnellement dans tous ses discours sur le coronavirus est moins intellectuelle, moins civilisée, pauvre en argumentativité et rapide comme une fusée ${ }^{4}$. Le seul critère de Vlad que cette formule ne remplit pas et qui détermine toute sa charge conflictuelle est le caractère privé de ces formes opposées à la polémique. C'est à dessein que Trump s'est attaqué publiquement, par le canal de la presse, à la nation chinoise. Cela est d'autant plus vrai que l'interrogation de la situation de communication ${ }^{5}$ nous fournit les arguments pour qualifier cette formule de comme telle. Ainsi, les critères de lecture de la situation de communication proposés par Maingueneau, renforcés par la lecture componentielle cidessus de Vlad, traduisent le caractère offensant de la parole de Trump qui n'a rien d'hypocoristique. Cela peut se prouver. Mais avant, plantons le décor par l'interrogation du contexte empirique de communication de la célèbre formule du Président américain.

Le coronavirus est une pandémie qui a surgi un 17 novembre 2019 dans la ville de Wuhan, en Chine centrale pour se répandre, en l'espace de quelques mois, à travers tout le globe terrestre. Parce qu'on n'a véritablement pas pris garde à l'épidémie quand elle enflammait la seule Chine et qu'on ne s'y est pas préparé en conséquence, les dégâts ne se sont pas fait attendre. C'est avec beaucoup d'amertume que les peuples et les nations vont découvrir leur fragilité et leurs faiblesses structurelles, jusquelà insoupçonnées. La pandémie du coronavirus détient le sombre mérite de remettre en cause des certitudes vieilles de plusieurs siècles et des croyances qu'on pensait incompressibles. La santé du monde est désormais en jeu et plus personne n'est à l'abri du danger de la pandémie extrêmement contagieuse, y compris les pays comme les États-Unis qui se croyaient invulnérables jusqu'à ce que leurs yeux se décillent sur la triste réalité. Dans la panique, le Président Trump qui a déjà des contentieux politiques, commerciaux et économiques avec la Chine va automatiquement accuser la nation rivale de n'avoir rien fait pour limiter le coronavirus à son seul territoire. Dans nombreux de ses tweets ${ }^{6}$, de ses

${ }^{4}$ Reprenons le propos de G. Dessons (2015: 92), nous dirons que la formule rapide de Trump donne "cette impression d'une flèche de pensée - sœur de la fusée baudelairienne - infligeant une blessure au sens commun ».

${ }^{5}$ Nous abordons ce concept dans le même sens que Maingueneau (2002: 14) qui le décrit ainsi: «En parlant de situation de communication, on considère en quelque sorte « de l'extérieur » la situation de discours dont le texte est indissociable ».

${ }^{6} \mathrm{La}$ vidéo mentionnée plus haut fait cas de ces tweets. 
conférences filmées et surtout dans ce discours écrit et rectifié quelques minutes avant sa prolation7, la formule «virus chinois » apparaît donc comme un argument ad nauseam ${ }^{8}$.

Le contexte d'énonciation qui entoure la profération ou l'écriture de cette formule ainsi présentée nous fournit le statut du locuteur, de ses allocutaires et du délocuté. Trump est le Président américain qui a engagé une guerre commerciale avec la Chine juste avant que n'éclate la pandémie de la Covid-19. Cette maladie apparaît donc comme une aubaine pour Trump de prendre le dessus dans le duel économique et commercial qui l'oppose au Président chinois Xi Jin Ping qui, jusque-là, ne s'est pas laissé intimidé par le géant américain. L'ethos de Trump révèle donc que c'est dans une posture trempée déjà dans la polémique qu'il a créé le concept de « virus chinois » et l'argument qu'il avance pour justifier son acte relève presque de l'hypocrisie:

Je dois l'appeler par d'où il vient. Il est venu de Chine donc je pense que ce terme est très précis.

On devine que ce message, dans lequel le délocuté est le virus de la Covid-19, est certes public, vu les circonstances de son émission et le canal de sa diffusion médiatique, mais qu'en réalité, c'est aux autorités chinoises avec qui Trump a des démêlées et des comptes à régler qu'il est adressé. Ce qui explique la grande frustration de ces autorités chinoises qui vont répliquer en dénonçant la légèreté du pouvoir américain et en l'accusant de brouiller les pistes de ses propres contradictions face à la pandémie émergente.

Les États-Unis ont plutôt essayé de calomnier les autres, de leur faire porter la responsabilité et de chercher des boucs émissaires. Cette approche n'est ni morale ni responsable, et non seulement elle ne contribue pas à la prévention de l'épidémie aux États-Unis eux-mêmes, mais elle ne facilite pas la lutte collective de la communauté internationale contre l'épidémie. Je veux ici exhorter une nouvelle fois les États-Unis à cesser immédiatement de politiser l'épidémie, à cesser de

\footnotetext{
${ }^{7}$ https://www.liberation.fr/direct/element/quand-trump-corrige-son-discours-a-la-mainpour-direvirus-chinois-plutot-que-coronavirus_110962/

${ }^{8}$ Dans le dictionnaire en ligne L'internaute, un argument qui dit que l'on fait quelque chose de façon obsessionnelle et répétitive (https://www.linternaute.fr/expression/languefrancaise/14277/ad-nauseam/).
} 
stigmatiser la Chine et à cesser de calomnier les autres pays. Ils devraient s'attacher à bien faire leur propre travail et à jouer un rôle constructif dans l'effort collectif de tous les pays pour lutter contre l'épidémie et maintenir la santé et la sécurité publiques mondiales. ${ }^{9}$

Cette réplique de l'autorité chinoise, s'il faut la considérer comme une légitime défense, n'en demeure pas moins aussi agressive et conflictuelle. Au vu des propos de Trump qui a accusé les chinois de n'avoir pas alerté suffisamment le monde du danger que constituait le virus apparu sur son sol, il ressort que la réaction des autorités de Pékin peut s'expliquer sans doute par la colère et la révolte devant les attaques répétées de Trump. À la limite, les propos du porte-parole de la Chine dans la vidéo contiennent des injures ${ }^{10}$ et il est sans doute plus discourtois que Trump, même s'il faut reprocher à ce dernier d'avoir été l'instigateur de la polémique. Les propositions suivantes sont très disqualificatrices de l'ethos états-unien: «Cette approche n'est ni morale ni responsable», « Ils devraient s'attacher à bien faire leur propre travail et à jouer un rôle constructif dans l'effort collectif de tous les pays ». Ici la double négation équivaut à dire, par euphémisme, que les autorités politiques des ÉtatsUnis sont immorales et irresponsables. Dans la deuxième expression, nous lisons bien une grave accusation portée contre les États-Unis présentés comme un pays laxiste et nihiliste. Mieux, dans la réplique de leur porteparole à ce qu'ils considèrent comme une agression verbale, les chinois sont, toute diplomatie mise à part, menaçants: " Je veux ici exhorter une nouvelle fois les États-Unis à cesser immédiatement de politiser l'épidémie, à cesser de stigmatiser la Chine et à cesser de calomnier les autres pays ». Il ne s'agit nullement d'une exhortation, mais plutôt d'une mise en garde que ne peut trahir l'emploi du modalisateur « immédiatement». Mais revenons à la formule polémique de Trump qui implante l'interlocution conflictuelle en tant que mise en jeu de la parole « infectée ».

Dans l'approche de la situation de communication, il y a, selon D. Maingueneau, le paramètre $\mathrm{du}$ " mode d'inscription dans la temporalité »

9 https://www.youtube.com/watch?v=BPpwLdIhBwM

${ }^{10}$ En guise de définition, on retient que «d'un point de vue énonciatif, l'injure est une interaction verbale d'un certain type, participant de la violence verbale, qui nécessite au moins la présence/participation de deux personnes et d'un objet; à savoir: l'injurieur, l'injure et l'injurié » (B. Fracchiolla, 2011: 2). 
(2002 : 14). La formule de Trump est certes ad nauseam du point de vue de la périodicité mais elle reste furtive, considération prise de sa durée dans le temps. En d'autres termes la vitesse ${ }^{11}$ de la parole de Trump est grande et $c^{\prime}$ est en cela que sa fonction perlocutoire est considérable. Elle est blessante et ne peut donc s'inscrire dans une éthique de la conversation ou de la communication. En réalité, en traitant le coronavirus de "virus chinois », Trump tombe sous le coup de cette proposition de G. Dessons : « En allant trop vite - c'est-à-dire en étant excessivement singulier - on s'expose à une sortie de route, une sortie hors du sillon tracé collectivement» $(2015$ : 94). Et la sortie de route de Trump a indisposé ses allocutaires directs que sont les chinois.

Il nous reste maintenant à déterminer le dernier paramètre susceptible de nous aider à caractériser la formule de Trump comme une agression verbale. Il s'agit de la finalité de cette formule. Si pour une seule fois, au cours de laquelle le Président américain a remplacé sur un coup de tête le terme en anglais «corona » par celui de «chinese» pour créer la formule "chinese virus » qui signifie en français "virus chinois », on note que toutes les autres occurrences de cette formule sont préméditées et expriment bien l'intentionnalité de l'auteur. Trump cherche à frustrer les chinois à qui il n'a pas pardonné la capacité à lui tenir la dragée haute. Il a donc fait ce que dit ici Paul Valery cité par G. Dessons : " usage du mot ou de l'acte pour son effet de choc instantané. Faible masse, grande vitesse » (2015 : 79). Dans le discours de Trump sur le coronavirus, la formule « virus chinois » serait comparée à une flèche lancée contre la Chine pour lui transpercer le cœur. Elle incarne toutes ces paroles antipathiques qui désocialisent et qui visiblement traduisent la panique face à la pandémie émergente plutôt que la recherche de solutions communes pour l'éradiquer. En clair, sous l'effet de la maladie, la parole politique s'est

11 Nous appréhendons ici la notion de vitesse dans la même perspective que Gerard Dessons (2015: 89), non pas comme un rapport entre une temporalité et une dimension, mais comme "un processus sémantique, c'est-à-dire, au sens d'Émile Benveniste, une signification-sujet, produite par l'instanciation d'un discours ». De ce point de vue, l'impression de rapidité qui peut s'éprouver à la lecture d'un texte provient du sentiment d'une parfaite coïncidence entre la production d'une pensée et son expression, avec l'idée que dans un tel discours, d'avantage qu'ailleurs, le langage n'est plus le truchement d'une pensée, le véhicule d'un raisonnement, mais qu'il est cette pensée même, ce raisonnement même » (Id., 79) 
infectée. Mais il semble que cette parole ne soit pas la seule à s'être troublée. Un dialogue entre les médecins Jean-Paul Mira et Camille Cocht, à propos de la vaccination contre la Covid-19, confirme cela.

\section{La corruption de la parole par les discours racistes et tendancieux dans la lutte contre la Covid-19}

À l'instar de la formule «virus chinois » de Trump que nous venons de décrire, il y a ces paroles qui de par leur teneur contribuent à diviser la société et à la dresser contre elle-même. Il s'agit, pour celles que nous voulons analyser ici, des paroles exclusivement racistes. Ces paroles, plus que la formule de Trump, donnent l'impression que la pandémie de la Covid-19 a complètement perverti les esprits au point de leur faire perdre raison. Et c'est parce qu'elles ont perdu la raison que des personnes disent des choses regrettables qui éjectent visiblement la parole du cadre de sa pratique idéale arrimée à celle du langage exalté ici par David Le Breton : « la possibilité du langage caractérise la condition humaine et fonde le lien social » (1997: 9). Le cas qui pourrait illustrer la parole raciste dans les rapports dialogiques et les liens sociaux est celui de deux spécialistes de médecine qui se sont autorisés sur la chaine de télévision LCI à parler au nom de la science qu'ils disent détenir pour sauver l'humanité. Nous retranscrivons les termes de la séquence du dialogue télévisé entre les deux spécialistes de médecine : la première réplique est de Jean-Paul Mira, chef de service à l'hôpital Cochin de Paris quant à la deuxième, elle, est de Camille Cocht, directeur de recherche de l'Institut national de la santé et de la recherche médicale (Inserm) :

"Si je peux être provocateur, est-ce qu'on ne devrait pas faire cette étude en Afrique, où il n'y a pas de masques, pas de traitements, pas de réanimation? Un peu comme c'est fait d'ailleurs pour certaines études sur le sida. Chez les prostituées, on essaye des choses parce qu'on sait qu'elles sont hautement exposées et qu'elles ne se protègent pas.

- Vous avez raison. On est d'ailleurs en train de réfléchir à une étude en parallèle en Afrique $»^{12}$

\footnotetext{
${ }_{12}$ Sources à consulter: https://www.ouest-france.fr/sante/virus/coronavirus/ coronavirus-un-vaccin-teste-en-afrique-la-sequence-de-lci-qui-indigne-lesreseaux-sociaux-6799161
} 
Cet échange a choqué le monde entier mais particulièrement deux cibles qui en sont le délocuté : les africains et les professionnelles du sexe. Les paroles des deux médecins marginalisent ces deux entités par racisme et par stigmatisation. Mais en quoi, ce dialogue entre Mira et Cocht est-il insultant pour leurs victimes ? La réponse s'articule en trois points essentiels qui sont: le mépris des locuteurs pour la race et l'homme, le complexe de supériorité qu'ils affichent ostensiblement et enfin l'insinuation et la diffamation de l'altérité.

À entendre les deux médecins, l'Afrique et les prostituées sont deux entités qui peuvent servir de cobayes pour les expérimentations scientifiques. Dans l'intervention du docteur Mira, un substantif est lourd de conséquence. C'est le mot «étude » répété par le locuteur, la preuve qu'il insiste sur un paradigme problématique pour lui comme pour sa cible, l'Afrique et les prostituées. Son propos identifie ouvertement le continent noir à un champ d'expérimentation et ses habitants, donc, à des cobayes à travers l'interrogation : "est-ce qu'on ne devrait pas faire cette étude en Afrique... ? ». Dans l'analogie qu'il crée entre la condition de l'Afrique et celle des professionnels du sexe, le médecin propose que le vaccin antiCovid l'Afrique soit expérimenté en Afrique «un peu comme c'est fait d'ailleurs pour certaines études sur le sida». Il ne faut pas s'y méprendre, même la prétérition "Si je peux être provocateur », en début de propos, ne peut euphémiser la gravité des propos des deux interlocuteurs. Au contraire, cette figure de rhétorique traduit le caractère prémédité et assumé de leur provocation ${ }^{13}$ dont ils n'ignoraient sans doute pas les probables retombés. Par leur attitude, on peut déduire la finalité ou l'intentionnalité de leur dialogue : dévaloriser ou vilipender une race et une catégorie sociale d'individus, en un mot, exprimer leur haine raciste. Il semble qu'aucune circonstance atténuante ne milite en leur faveur. D'ailleurs, à ce mépris pour la race et l'homme, s'ajoute la preuve que l'interlocution des deux professionnels de la santé révèle chez eux deux

https://www.youtube.com/watch?v=ST5Wic8_6Y

${ }_{13}$ Sur la provocation, nous (A. Gounougo, 2017) avons produit ailleurs une contribution dans laquelle nous soutenions qu'en dépit de la grande similitude entre elles, il ne fallait nullement confondre la provocation, reposant sur une axiologie négativiste, avec la liberté d'expression, une qualité d'être au monde nécessairement vertueuse. Sur cette base, les médecins Mira et Cocht ont provoqué et choqué leurs victimes (l'Afrique et les prostituées) et leur attitude ne saurait se justifier par un quelconque droit à la liberté d'expression. 
autres traits de comportement, à savoir le complexe de supériorité qu'ils affichent ostensiblement et l'insinuation doublée d'une diffamation.

L'image que les deux médecins français, en effet, donnent de l'Afrique et des prostituées est négative. Il y a un esprit manichéen qui animent les deux interlocuteurs et le schème directeur de leur système idéologique peut, à l'infini, s'évaluer en termes dialectiques : le mal/le bien, la pauvreté/la richesse, l'ignorance/la pauvreté, l'Afrique/l'Occident, le laboratoire/le chercheur, etc. Sur la base de cette description, il est possible de répertorier les termes de la contradiction dialectique dont la conversion des contraires, dans l'esprit des médecins est en faveur du second pôle de la contradiction, c'est-à-dire, celui qu'ils incarnent. Deux enthymèmes peuvent être formulés ici, qui sont :

L'Afrique n'a pas les moyens pour contrer la Covid-19. Or l'occident possède ces moyens. Donc l'Afrique est inférieure à l'Occident.

Les prostituées exposées au sida ne se protègent pas. Les personnes non exposées au sida se protègent. Donc les prostituées sont inférieures aux autres personnes.

La conséquence du fonctionnement de la conscience de par de tels schèmes est que les deux médecins croient avoir le droit de faire disposer de la vie des africains et des prostituées par autrui dès l'instant où ces êtres représentent l'échantillon idéal pour leurs recherches. Partant de là, nul besoin d'être docte pour comprendre que le dialogue entre les deux médecins repose sur des contrevérités : l'Afrique n'est pas ce qu'ils croient qu'elle est, les prostituées ne sont pas des êtres inférieurs. Les réactions qui ont fusé de partout dans le monde, pendant et après leur entretien, montrent que Mira et Cocht ont tenus des propos racistes qui n'ont laissé personne indifférent, surtout les africains. Ce n'est pas Samuel Eto'o fils ${ }^{14}$ qui nous contredira:

Fils de P...

Vous n'êtes que de la MERDE,

N'est-ce pas l'Afrique est votre terrain de jeu...

Face à cette réplique du footballeur africain, qui n'est d'ailleurs pas le seul athlète de renom à avoir répondu aux deux professeurs de médecine, il ressort clairement que la parole s'est véritablement débridée.

${ }^{14}$ https://www.facebook.com/setoo99/posts/3662509987153601

Dialogos • Vol. XXII No. 38/2021 
Elle n'est plus portée par une éthique. En cela, nous disons que la pandémie de la Covid-19, qui panique tout le monde et le pousse à ne plus raisonner, a infecté la parole. Celle-ci est discourtoise et antipathique. La parole raciste des professeurs Mira et Cocht a eu sa réplique de taille qui, elle aussi n'est pas propre et exempte de reproches. Le footballeur africain a tout simplement insulté les médecins en guise de réaction à leur manque de respect. Les expressions «fils de $\mathrm{P}$... » et «vous êtes de la merde » sont deux métaphores dépréciatives en tant qu'elles sont des insultes lancées contre les allocutaires. Le fait d'employer l'apocope «p...» pour faire preuve de pudeur n'atténue en rien, en réalité, la teneur de l'injure d'Eto'o Fils car aucun locuteur de langue française n'ignore que cette figure de construction désigne le substantif «putes». En choisissant de signaler seulement ce substantif par son premier phonème au lieu de le proférer dans toute son entièreté morphologique, Eto'o Fils l'a rendu plus bruyant au point d'attirer sur lui l'attention des récepteurs, non dupes, de son message. Quand on applique les sèmes permettant de distinguer la polémique des autres formes de conflits de la parole qui lui ressemblent, selon la proposition de Vlad citée plus haut, nous avons, pour les propos de l'athlète africain, les éléments suivants : [- activité intellectuelle], [- civilité], [+ argumentativité faible], [- durée] », avec une exception pour le sème [+ caractère privé] qui se justifie par le canal de diffusion desdits propos. Au dialogue des deux professionnels de la santé, s'applique aussi ces mêmes sèmes, ce qui prouve aisément que la parole, dans sa fonction irénique de socialisation a été tout simplement corrompue, sous la panique de la pandémie qui est ainsi l'objet de violentes controverses plutôt que de la recherche de solutions sanitaires.

\section{La parole conspirationniste : une autre parole pervertie pour expliquer la Covid-19}

La parole conspiratoire nous intéresse dans le cadre de cette contribution parce qu'elle a un caractère naturel qui nous oblige à remettre en cause sa fonction de socialisation. Pour appréhender ce caractère il est nécessaire de définir le conspirationnisme. C'est à P. Zawadzki que nous empruntons la définition suivante du concept. Pour le chercheur, le conspirationnisme désigne : «la croyance en l'existence d'un plan illégal organisé volontairement par des individus mal intentionnés, et l'usage de cette croyance pour expliquer certains faits ou le cours de l'histoire » (2010 : 
44). Et quelques traits fondamentaux de cette croyance sont dans cette proposition d'E. Danblon et L. Nicolas :

Ainsi, le plus souvent, l'ethos conspirationniste présente un mélange apparemment incohérent d'expertise (blouse blanche, longue liste de titres universitaires, etc.) et de marginalité (construction d'un ethos "alternatif ", à la marge des institutions officielles). Quant au pathos, il suscite un mélange de ressentiment et d'indignation. Il cherche à rassurer (dévoilement, identification de l'ennemi) autant qu'à inquiéter (présentation d'un système occulte et gigantesque). Enfin, pour ce qui est du logos, on trouve une hypertrophie de la critique qui confine, à l'occasion, à la ratiocination (...) en même temps qu'une habitude peu compatible avec les principes de la critique de s'immuniser contre toute tentative de réfutation (2012 : 35-36)

La parole qui se construit dans un tel environnement de suspicion ou $\mathrm{du}$ moins cette parole que le conspirationniste profère en vue d'expliquer sa vision du monde dans lequel il refuse de rester passif pour ne pas le subir, n'est pas toujours une parole empathique et socialisante. Son caractère suffisant, fermé et le plus souvent aversif fait que la parole conspiratoire tombe dans de grands excès qui la corrompent nécessairement. Quel est donc ce locuteur qui « en s'affranchissant de la charge de la preuve, (...) immunise son discours contre toute critique » (Reichstadt, $2015: 3$ ) et cherche à « minimiser sinon à nier la part de hasard, d'imprévisible, de fortuit qui intervient dans la réalité (...), cherchant des signes là où il n'y en a pas et conférant un sens explicatif à des liens qui, pour être objectifs, n'en sont pas nécessairement signifiants »? (Id.). C'est ce type de parole, qui aime la critique des autres et abhorre la sienne, qui sert à Kemi Seba ${ }^{15}$ pour non seulement répondre aux deux racistes cachés derrière leur statut de médecins mais également pour régler d'autres comptes portant sur des topiques éloignées de celle de la Covid-19. Dans la conférence $^{16} \mathrm{du}$ leader anti-Françafrique, les expertises s'entremêlent, les thématiques fusent, la ratiocination est à l'œuvre, le tout donnant

${ }^{15}$ Kemi Seba est un activiste africain, de nationalité franco-béninoise. Il est le chef de file du mouvement politique «Urgences panafricanistes » et est connu pour ses prises de positions tranchées contre l'impérialisme, le néocolonialisme et l'oligarchie occidentale mais également contre des régimes africains qu'ils suspectent d'être de connivence avec l'Occident pour piller l'Afrique.

${ }^{16} \mathrm{https} / / /$ www.youtube.com/watch? $\mathrm{v}=\mathrm{ZkWqO} 9 \mathrm{D} 4 \mathrm{rr} 0$ 
l'impression d'un savoir ou d'une parole absolue qui est ainsi fermée à toute critique. Nous allons, dans le tableau qui suit, relever les preuves qui s'intriquent dans le discours de Kemi Seba sur la Covid-19, en vue d'analyser par la suite l'ethos du leader panafricaniste qui, sans doute, inquiète plus qu'il ne veut rassurer.

\begin{tabular}{|c|c|c|}
\hline THÈSE & ARGUMENTS & PREUVES \\
\hline \multirow{5}{*}{ 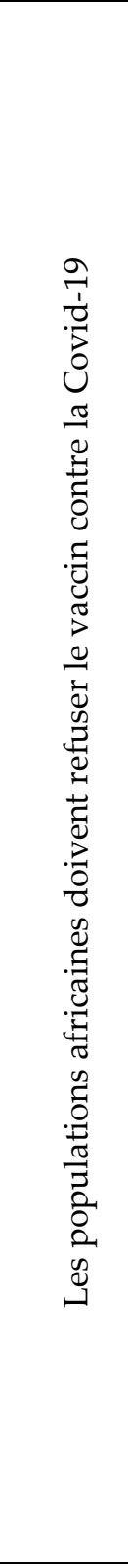 } & $\begin{array}{l}\text { Les tests de vaccination } \\
\text { contre la Covid-19 sont } \\
\text { un enjeu de lutte de } \\
\text { classes }\end{array}$ & $\begin{array}{l}\text { «ce n'est pas une question de noir contre } \\
\text { blanc de blancs contre noir c'est une question } \\
\text { de puissants contre des pauvres » }\end{array}$ \\
\hline & $\begin{array}{l}\text { Des lobbies s'enrichissent } \\
\text { sur le dos des plus } \\
\text { pauvres }\end{array}$ & $\begin{array}{l}\text { "pour comprendre exactement la manière } \\
\text { dont ces grands groupes pharmaceutiques } \\
\text { opèrent, il faut savoir que dans toute guerre, } \\
\text { il y a toujours deux lobbies qui sont les } \\
\text { premiers à se remplir les poches et à se faire } \\
\text { de très très très grandes fortunes : c'est le } \\
\text { lobby des armes, dans les guerres d'accord et } \\
\text { la banque» }\end{array}$ \\
\hline & $\begin{array}{l}\text { Le lobby pharmaceutique } \\
\text { est celui qui mène la } \\
\text { guerre bactériologique } \\
\text { contre les pauvres }\end{array}$ & $\begin{array}{l}\text { «le lobby des armes se nomme le lobby } \\
\text { pharmaceutique, lobby pharmaceutique qui } \\
\text { travaille à partir des plantes et va faire un } \\
\text { certain nombre d'expériences pour qu'à } \\
\text { partir des plantes ils puissent créer des } \\
\text { médicaments qu'ils pourront vendre à prix } \\
\text { qui dépasse la raison à nos états» }\end{array}$ \\
\hline & $\begin{array}{l}\text { La solution de la Covid- } \\
19 \text { est naturellement dans } \\
\text { les plantes }\end{array}$ & $\begin{array}{l}\text { «je dis bien aucune maladie qui apparaît sur } \\
\text { cette terre sans qu'il y ait une réponse } \\
\text { adéquate qui sont données à travers mes } \\
\text { plantes et ça c'est important qu'on puisse le } \\
\text { préciser de la manière la plus crue et la } \\
\text { manière la plus simple possible» }\end{array}$ \\
\hline & $\begin{array}{l}\text { La vaccination en } \\
\text { Afrique est un crime } \\
\text { contre l'humanité }\end{array}$ & $\begin{array}{l}\text { «c'est un crime contre l'humanité ce genre } \\
\text { de choses-là. Ces gens-là prennent nos } \\
\text { populations comme des cobayes, ces gens-là } \\
\text { prennent nos populations comme des } \\
\text { cimetières à ciel ouvert en tant que tels, sur } \\
\text { lesquels ils peuvent se permettre de tout } \\
\text { faire. Le but, c'est bien évidemment, comme } \\
\text { on le rappelle de manière systématique, la } \\
\text { réduction de la population » }\end{array}$ \\
\hline
\end{tabular}




\begin{tabular}{|c|c|c|}
\hline THÈSE & ARGUMENTS & PREUVES \\
\hline & $\begin{array}{l}\text { Les médicaments } \\
\text { pharmaceutiques ne sont } \\
\text { pas bons car dangereux } \\
\text { pour la santé des } \\
\text { africains }\end{array}$ & $\begin{array}{l}\text { "ces vaccins ont des effets secondaires pires } \\
\text { que la maladie même, initiale, mais il vous } \\
\text { les administrent et les ont administrés sur } \\
\text { bon nombre de populations africaines" }\end{array}$ \\
\hline & $\begin{array}{l}\text { Des complots visent la } \\
\text { destruction de l'Afrique }\end{array}$ & $\begin{array}{l}\text { «il y a une guerre de l'oligarchie financière } \\
\text { occidentale qui travaille en concubinage } \\
\text { incestueux avec la population élitiste, } \\
\text { l'oligarchie africaine» }\end{array}$ \\
\hline & $\begin{array}{l}\text { Les élites africaines sont } \\
\text { le mal de leurs peuples }\end{array}$ & $\begin{array}{l}\text { «les populations aujourd'hui ont compris } \\
\text { que les élites se délitent et que les élites sont } \\
\text { les pires ennemies en réalité de l'intérêt des } \\
\text { peuples et c'est pour ça que la question de la } \\
\text { souveraineté populaire en ce début de } \\
\text { vingtième siècle doit revenir plus que } \\
\text { jamais» }\end{array}$ \\
\hline
\end{tabular}

Ce tableau donne un aperçu de la complexité du sujet abordé par Kemi Seba, ou du moins c'est lui-même qui en donne une telle qualité. La parole du leader des Urgences panafricanistes ${ }^{17}$, du fait de la thèse qu'elle véhicule, dépeint un ethos complexe pour ne pas dire éclaté. Le locuteur est à la fois un spécialiste de santé publique, un chercheur en médecine, un homme de droit, mieux un justicier, un sonneur d'alerte, un économiste doublé d'un financier, un historien, un marxiste, etc. Ce trop-plein de savoir fait de lui un sachant au discours incontestable et c'est ce caractère absolu de l'argumentation, qui ne laisse aucune place à la contradiction, qui nous pousse à considérer cette parole de Kemi Seba comme une théorie complotiste $^{18}$ et donc comme une autre forme de parole pervertie qui contribue plus à exacerber, par la suspicion, les conflits entre les hommes et non à leur procurer la paix mondiale comme elle peut penser le faire. Ainsi,

${ }^{17}$ Urgences panafricanistes est l'ONG que dirige Kemi Seba.

${ }^{18}$ Cette définition de L. Nicolas (2014: 1) de la théorie complotiste confirme notre décision de classer ce discours de Kemi Seba comme tel: ces théories (1) recueillent et assemblent des événements élevés au statut de faits (Dominicy, 2010), dans le but (2) d'apporter la preuve que ces faits sont nécessairement liés entre eux, (3) parce qu'ils résultent d'une cause unique, c'est-à-dire (4) d'un complot dont ils témoignent, et (5) au sein duquel les participants agissent conformément à une nature profonde qui les détermine. 
la volonté de Kemi Seba de « douter de tout pour ne pas douter du tout $»^{19}$ finit par l'exposer au doute des autres. Le fait de limiter exclusivement son argumentation à sa face pars destruens pour faire fi de la pars construens, la parole du panafricaniste peut se voir opposer le corax que $O$. Reboul définit en ces termes: "argument montrant qu'une chose est si vraisemblable qu'elle en devient invraisemblable » $(1991: 237)$. Tout ce que dit Kemi Seba peut être vrai mais l'excès avec lequel il le dit peut faire douter de son doute personnel, ce qui est susceptible de limiter la portée de sa parole. En d'autres termes, à force de diaboliser ceux qu'il désigne sous les concepts d'oligarchies, de lobbies, de comploteurs, de population élitiste, etc. sans possibilité de leur accorder ne serait-ce qu'un micron de positivité, le patron des Urgences panafricanistes s'est vu affublé du titre, semble-t-il, peu honorable de complotiste que d'ailleurs il récuse dans sa vidéo : « il y a quelques temps, peu de jours encore, certains parmi les nôtres dans le showbiz, des entités très fragiles, de parler de complotisme lorsque l'on disait qu'il y a une guerre qui est menée contre les populations, contre la population africaine (sic) » ou encore « c'est Jacques Attali qui disait aussi, rappelons-le, il y a quelques années, que si le gouvernement mondial et, ça ce n'est pas du complotisme là encore, c'est vérifiable et je vous demande, je vous en supplie, je demande à tous de vérifier ».

Nulle part dans le discours de Kemi Seba, l'on note une qualité chez tous ceux qu'il regroupe sous l'appellation d'oligarchies occidentales ou de lobbies si bien que le professeur Didier Raoult, le seul qui semble s'inscrire dans la même vision explicative du monde que les panafricanistes, n'est seulement évoqué que de façon furtive tant l'exception confirme la règle: « tout le monde parle du professeur Raoult qui fait un travail honorable en France et tant mieux pour lui ». Dans cette formule le brin d'honnêteté affiché par le locuteur a été de très courte durée car balayé rapidement par cette expression restrictive " tant mieux pour lui ». L'apport de ce professeur à la cause de la science n'est donc pas utile à la cause africaine mais seulement à la sienne, surtout que des médecins africains tels que Valentin Agon existent et font sans doute mieux que lui dans le domaine de la recherche scientifique :

19 Le dernier segment du titre de la réflexion de L. Nicolas qui précède: "L'évidence du complot: un défi à l'argumentation. Douter de tout pour ne plus douter du tout ». 
il y a aussi de l'autre côté, en Afrique, un professeur, le professeur Valentin Agon qui a travaillé sur un médicament qui se nomme Apivirine, qui a été testé sur un certain nombre de patients au Burkina Faso et qui donne pour l'instant de très bons résultats.

Comme nous pouvons le constater, un rapprochement existe entre les trois discours de Kemi Seba, Donald Trump et les deux médecins qui ont défrayé la chronique avec leurs propos racistes sur la chaîne de télévision LCI. Aussi, ne peut-on boucler notre réflexion sur la parole complotiste de Kemi Seba pour en révéler la nature conflictuelle sans évoquer le relâchement d'un certain lexique employé par ses soins et qui traduit toute la charge de l'argument ad hominem : " visage de la manière la plus pétrifiée », " couillonneurs», « usuriers», «crime contre l'humanité », «concubinage incestueux», «serment d'hypocrite », « clochardisation sanitaire ». Visiblement ces lexèmes ne sont pas employés pour appeler les comploteurs et leurs victimes à l'entente et la communication apaisée. Toutes ces expressions fonctionnent comme des arguments ad hominem, en ce sens que Kemi Seba adopte une attitude de polémiqueur en s'en prenant à la personne de chacun de ses adversaires. D'ailleurs, certaines de ces expressions se situent à la limite des injures et ne vont pas dans le sens du dialogisme apaisé. Au contraire, elles renforcent le sentiment de la polémique, mieux du conflit qui oppose l'orateur conspirationniste qu'est Kemi Seba aux présumés comploteurs.

\section{Conclusion}

$\mathrm{Au}$ terme de cette analyse de la rhétorique des trois discours de notre corpus, il ressort que la Covid-19, du fait de la panique qu'elle a provoquée dans la société, a fait découvrir chez de nombreuses personnes des comportements excessifs et empathiques. Ce sont les cas de personnes qui ont communiqué sur le coronavirus tels que Donald Trump, les deux médecins sur LCI et Kemi Seba de l'ONG «Urgences panafricanistes ». Leurs discours ont montré que la parole peut être source de conflits, à réception, selon qu'elle se fonde sur telle ou telle posture argumentative qui privilégie les oppositions et antagonismes. La rhétorique de la Covid19, du point de vue de ces discours, a bel et bien une tonalité polémique et $c^{\prime}$ est pourquoi nous pouvons affirmer que la pandémie a, d'une certaine façon, compliqué le dialogue entre certains acteurs sociaux qui, s'ils ne 
s'accusent pas mutuellement, tiennent des discours qui les opposent les uns aux autres. Dans tous les cas, la parole "infectée », telle que nous l'avons abordée à travers les propos de Donald Trump, des deux médecins et de Kemi Seba, est source d'interaction conflictuelle dans laquelle l'ironie et la satire apparaissent comme des stratégies argumentatives pour déstabiliser les autres ou pour banaliser leur sort. C'est dire, à tout prendre, que la parole qui blesse, désocialise et qui crée plus de problèmes qu'elle n'en résout, est malade au même titre que les corps physiques qui la profèrent. Dit autrement, La pandémie ne fait pas que détruire seulement le corps humain, elle pousse les hommes à dire des paroles graves et lourdes de conséquences ou, pour prendre à notre avantage cette assertion du poète français Baudelaire ${ }^{20}$, la Covid-19 pousse les hommes à laisser sortir des paroles confuses qui dressent la société contre elle-même.

\section{Références bibliographiques}

1. AMOSSY, Ruth (2012), L'argumentation dans le discours, Armand Colin, Paris

2. BRETON, Philippe (2007), Éloge de la parole, Éditions La Découverte, Paris

3. DANBLON, Emmanuelle, NICOLAS, Loïc (2012), Rhétorique et topique de la conspiration, [en ligne] https://www.researchgate. net/publication/312189788

4. DESSONS, Gerard (2015), La voix juste, Éditions Manucius, Paris

5. DOMINICY, Marc (2010), «Les sources cognitives de la théorie du complot. La causalité

6. et les "faits" ", Les rhétoriques de la conspiration, Emmanuelle Danblon et Loïc Nicolas (dir.), CNRS Éditions, Paris

7. DUCROT, Oswald et TODOROV, Tzvetan (1972), Dictionnaire encyclopédique des sciences du langage, Seuil, Paris.

8. FRACCHIOLLA, Béatrice (2011), «Injure», Dictionnaire de la Violence de Michela Marzano, PUF, Paris

${ }^{20}$ Dans son poème symbolique «Correspondances », ce dernier parle de « confuses paroles» pour désigner le caractère anagogique des symboles qui fondent l'existence. Nous, nous parlons de «paroles confuses » pour décrire les paroles embrouillées qui relèvent des excès et que rien ne justifie dans cette période trouble de pandémie, période dans laquelle l'essentiel devait être la recherche de solutions pour l'endiguer. 
9. GOUNOUGO, Aboubakar (2017), La provocation dans les médias modernes : quels enjeux, quels envers?, Sciences, Langage et Communication, Volume $1, \mathrm{~N}^{\circ} 2$.

10. LE BRETON, David (1977), Du silence, Éditions Métailié, Paris

11. MAINGUENEAU, Dominique (2002), Situation d'énonciation, situation de communication, La lingüística francesa en el nuevo milenio, lleida.

12. MOESCHLER, Jacques et REBOUL, Anne (1994), Dictionnaire encyclopédique de pragmatique, Seuil, Paris

13. NICOLAS, Loïc "L'évidence du complot : un défi à l'argumentation. Douter de tout pour ne plus douter du tout ", Argumentation et Analyse du Discours [Online], 13 | 2014, Online since 14 October 2014, connection on 23 September 2019. URL : http://journals.openedition .org/aad/1833 ; DOI : 10.4000/aad.1833

14. REBOUL, Olivier (1991), Introduction à la rhétorique, PUF, Paris

15. REICHSTADT, Rudy (2015), " conspirationnisme : un état des lieux », Note $\mathrm{n}^{\circ}$ 11, Fondation Jean-Jaurès, Observatoire des radicalités politiques

16. VLAD, Daciana, (2010), La polémique-une forme particulière de communication conflictuelle, Philologia, LV, 1, Editura Presa Universitarã Chujeanã

17. ZAWADZKI, Paul, (2010), «Historiciser l'imaginaire du complot. Note sur un problème

18. d'interprétation ", Les rhétoriques de la conspiration, Emmanuelle Danblon et Loïc Nicolas (dir.), CNRS Éditions, Paris 